\title{
SYMMETRY OF GLOBAL SOLUTIONS TO A CLASS OF FULLY NONLINEAR ELLIPTIC EQUATIONS IN 2D
}

\author{
D. DE SILVA AND O. SAVIN
}

\begin{abstract}
We prove that entire bounded monotone solutions to fully nonlinear equations in $\mathbb{R}^{2}$ of the form $F\left(D^{2} u\right)=f(u)$ are one-dimensional, under appropriate compatibility conditions for $F$ and $f$. In the particular case when $F=\Delta$ and $f(u)=u^{3}-u$, our result gives a new (non-variational) proof of the well known De Giorgi's conjecture.
\end{abstract}

\section{INTRODUCTION}

Let $u \in C^{2}\left(\mathbb{R}^{n}\right)$ be a solution to the following problem on $\mathbb{R}^{n}$,

$$
\triangle u=u^{3}-u, \quad|u| \leq 1, \quad u_{x_{n}}>0
$$

In 1978 De Giorgi made the conjecture that all the level sets of $u$ are hyperplanes, at least if $n \leq 8$.

This conjecture has been widely investigated. It was first proved for $n=2$ by Ghoussoub and Gui [3], and for $n=3$ by Ambrosio and Cabre [1]. Finally, in [4] the second author proved that the conjecture is true in any dimension $n \leq 8$, under the natural hypothesis

$$
\lim _{x_{n} \rightarrow \pm \infty} u\left(x^{\prime}, x_{n}\right)= \pm 1
$$

In this paper, we prove this conjecture for a more general class of fully nonlinear elliptic equations in 2D. The techniques used in the previous cited results, exploit the variational formulation of (1.1). In our case, there is no equivalent variational formulation for the problem, and we rely exclusively on the maximum principle and on elementary geometric considerations to prove the conjecture.

We consider the fully nonlinear reaction-diffusion equation in $\mathbb{R}^{2}$ :

$$
F\left(D^{2} u\right)=f(u)
$$

where $F$ is uniformly elliptic with ellipticity constants $\lambda, \Lambda, F(0)=0$, and

$$
f \in C^{1}([-1,1]), \quad f( \pm 1)=0, \quad f^{\prime}(-1)>0, \quad f^{\prime}(1)<0,
$$

$$
f \text { has only one zero in }(-1,1) \text {. }
$$

\footnotetext{
${ }^{1}$ Portions of this work were carried out while the first author was visiting MSRI at Berkeley. The authors would like to thank this institution for its hospitality.
} 
Furthermore, we assume that there exists an increasing function $g_{0}: \mathbb{R} \rightarrow[-1,1]$ (one dimensional solution) such that

$$
\lim _{t \rightarrow \pm \infty} g_{0}(t)= \pm 1
$$

and $g_{0}$ solves the equation in all directions $\xi$, that is

$$
F\left(D^{2}\left(g_{0}(x \cdot \xi)\right)\right)=f\left(g_{0}(x \cdot \xi)\right)
$$

for every unit vector $\xi \in \mathbb{R}^{2}$.

Under such assumptions, we prove that entire, monotone and bounded solutions to (1.2) are one-dimensional functions. Precisely, our main Theorem reads as follows.

Theorem 1.1. Let $u \in C\left(\mathbb{R}^{2}\right)$ be a viscosity solution to (1.2), such that $|u| \leq 1$ and $u_{x_{2}}>0$. Then, all level sets of $u$ are hyperplanes.

We remark that the assumption (1.4) is not essential for the validity of our result.

The main step in our proof consists in showing that if two balls at unit distance from the zero level set of $u$ are contained in the negative side, then their convex hull is also contained in the negative side. To achieve this, we use the maximum principle together with appropriate radially symmetric supersolutions. Then, from this fact, we deduce that $\{u=0\}$ is contained in a strip of arbitrarily small width, which implies the desired result.

The paper is organized as follow. In Section 2, we introduce some notation and tools which we will use in the body of the proof. In Section 3, we prove our main Theorem.

\section{Preliminaries AND MAIN TOOLS}

In this section we introduce certain notation and tools which we will be using throughout the paper.

A disk in $\mathbb{R}^{2}$, centered at $x_{0}$ and with radius $R$ will be denoted by $B\left(x_{0}, R\right)$. Also, for any two points $P_{1}, P_{2} \in \mathbb{R}^{2}$, we denote by $\overline{P_{1} P_{2}}$ the line segment joining them. If $\nu$ is a unit vector (direction) in $\mathbb{R}^{2}$, then $\nu^{\perp}$ denotes the perpendicular direction to $\nu$.

Let $g: I \rightarrow \mathbb{R}, I$ interval in $\mathbb{R}$ containing 0 , be such that $g^{\prime}>0$ and $g(0)=0$. Then we associate with $g$ a function $h(s)$ defined by the following property

$$
g^{\prime}(t)=\sqrt{2 h(g(t))}
$$

A straightforward computation gives $g^{\prime \prime}(t)=h^{\prime}(g(t))$. Set,

$$
H(s)=\int_{0}^{s} \frac{1}{\sqrt{2 h(t)}} d t,
$$

then $\left(H(g(t))^{\prime}=1\right.$, and hence

$$
g(t)=H^{-1}(t)
$$


Denote by $h_{0}, H_{0}$ the corresponding functions for the one-dimensional solution $g_{0}$, defined in Section 1 (without loss of generality we assume $g_{0}(0)=0$ ). If $g$ as above is defined for $|t| \leq R / 2$ then

$$
v_{R, g}(x):=g(|x|-R)
$$

satisfies (in the appropriate system of coordinates),

$$
\begin{gathered}
F\left(D^{2} v\right)=F\left(\begin{array}{cc}
g^{\prime \prime} & 0 \\
0 & g^{\prime} /|x|
\end{array}\right)=F\left(\begin{array}{cc}
h^{\prime}(g) & 0 \\
0 & \sqrt{2 h(g)} /|x|
\end{array}\right) \leq \\
F\left(\begin{array}{cc}
h_{0}^{\prime}(g) & 0 \\
0 & 0
\end{array}\right)+\frac{2 \Lambda}{R} \sqrt{2 h}+\max \left\{\lambda\left(h^{\prime}-h_{0}^{\prime}\right), \Lambda\left(h^{\prime}-h_{0}^{\prime}\right)\right\}= \\
f(v)+\frac{2 \Lambda}{R} \sqrt{2 h}+\max \left\{\lambda\left(h^{\prime}-h_{0}^{\prime}\right), \Lambda\left(h^{\prime}-h_{0}^{\prime}\right)\right\},
\end{gathered}
$$

where the equality

$$
F\left(\begin{array}{cc}
h_{0}^{\prime}(s) & 0 \\
0 & 0
\end{array}\right)=f(s)
$$

follows from the assumption (1.5).

Thus, if

$$
h^{\prime}(s)+\frac{C(\lambda, \Lambda)}{R} \sqrt{2 h(s)}<h_{0}^{\prime}(s)
$$

then $v_{R, g}$ is a strict supersolution on the $\left\{v_{R, g}=s\right\}$ level set.

Analogously, set $w_{R, g}(x):=g(R-|x|)$, a similar computation shows that for

$$
h^{\prime}(s)-\frac{c(\lambda, \Lambda)}{R} \sqrt{2 h(s)}<h_{0}^{\prime}(s)
$$

$w_{R, g}$ is a strict supersolution on the $\left\{w_{R, g}=s\right\}$ level set.

In the next Lemma we prove the existence of a radially symmetric supersolution, whose profile is a perturbation of the one-dimensional solution. The same result is proved in detail in [4]. For completeness, we present its proof.

Lemma 2.1. There exists a function $g_{R}$ that is constant for $|t|>R / 2$, such that $g_{R}(|x|-R)$ is a supersolution everywhere except on the 0 level set. Moreover, the associated function $H_{R}:\left[-1+e^{-c R}, 1\right] \rightarrow \mathbb{R}$, satisfies

$$
-\frac{C}{R} \log (1-|s|) \geq H_{0}(s)-H_{R}(s) \geq 0, \text { for }|s|<1-e^{-c R / 2} .
$$


Proof. Let $s_{R}=e^{-c R}$ and define the following function $h_{R}$ with a jump discontinuity at 0 ,

$$
h_{R}(s)= \begin{cases}h_{0}(s)-h_{0}\left(s_{R}-1\right)-\frac{C}{R}\left[(1+s)^{2}-s_{R}^{2}\right] & \text { for } s_{R}-1 \leq s \leq 0, \\ h_{0}(s)+h_{0}\left(s_{R}-1\right)+\frac{C}{R}\left(1-s+s_{R}\right)(1-s) & \text { for } 0<s \leq 1 .\end{cases}
$$

According to (2.4), we need to show that

$$
h_{R}^{\prime}(s)+\frac{C(\lambda, \Lambda)}{R} \sqrt{2 h_{R}(s)}<h_{0}^{\prime}(s)
$$

for all $s \neq 0$.

From (2.3) we obtain that $h_{0}^{\prime}$ is proportional to $f$. Therefore, using (1.3) we get that,

$$
h_{0}^{\prime}(s) \sim c(s+1), \quad \text { near } s=-1, \quad h_{0}^{\prime}(s) \sim c(s-1), \quad \text { near } s=1
$$

Thus,

$$
\begin{aligned}
& h_{R}(s) \sim c\left[(1+s)^{2}-s_{R}^{2}\right], \quad s \in\left[s_{R}-1,0\right] \\
& h_{R}(s) \sim c\left[(1-s)^{2}+s_{R}^{2}\right], \quad s \in[0,1] .
\end{aligned}
$$

Then, (2.8) and the corresponding estimates for $H_{R}$ follow from straightforward computations.

\section{Proof of Theorem 1.1.}

In this section, we prove Theorem 1.1. We start by assuming that,

$$
\lim _{x_{2} \rightarrow \pm \infty} u\left(x_{1}, x_{2}\right)= \pm 1 \text {. }
$$

At the end of the section, we then prove that (3.1) must necessarily be satisfied by our solution $u$.

We start by proving the main tool in our proof, which is the following statement. Here and henceforth, the constant $C$ depends only on $\lambda, \Lambda$, on the modulus of continuity of $f^{\prime}$, and on $f^{\prime}( \pm 1)$.

Proposition 3.1. Assume $B\left(y_{i}, R\right) \subset\{u<0\}, i=1,2$. Then the convex hull generated by

$$
B\left(y_{i}, R-C \frac{\log R}{R}\right), i=1,2
$$

is included in $\{u<0\}$.

The proof of Proposition 3.1 is divided in several steps. We start by proving the following Harnack-type inequality for the level sets. Here and in the next Lemma, the function $g_{R}$ will denote the supersolution from Lemma 2.1. 
Lemma 3.2. If $B\left(y_{0}, R\right) \subset\{u<0\}$ then

$$
u(x) \leq g_{R}\left(\left|x-y_{0}\right|-R\right) .
$$

Moreover, for any $M>0$ and $R \geq C(M)$, if $\bar{B}\left(y_{0}, R\right)$ touches $\{u=0\}$ at $x_{0}$ then there exists a ball of radius $M$ at distance $1 / M$ from $x_{0}$ included in $\{u>0\}$.

Proof. The first part of our statement follows by sliding $g_{R}\left(\left|x-y_{0}\right|-R\right)$ from $-\infty$. For the second part, let

$$
\tilde{v}(x):=g_{0}\left(\left|x-y_{0}\right|-R+\frac{C_{1}(M)}{R}\right) .
$$

Then, using (2.6), we derive that for ||$x-y_{0}|-R| \leq 4 M$, we have

$$
\tilde{v}(x) \geq g_{R}\left(\left|x-y_{0}\right|-R\right),
$$

if $C_{1}$ is chosen large. Thus,

$$
\tilde{v}(x) \geq u(x) \text { in } B\left(x_{0}, 4 M\right) \text { and } \tilde{v}\left(x_{0}\right)-u\left(x_{0}\right) \leq \frac{C_{2}(M)}{R} .
$$

Following the computation in (2.2), we obtain

$$
f(\tilde{v}) \leq F\left(D^{2} \tilde{v}\right) \leq f(\tilde{v})+\frac{C}{R} \sqrt{2 h_{0}(\tilde{v})} \leq f(\tilde{v})+\frac{C_{3}(M)}{R} .
$$

Therefore,

$$
\left|F\left(D^{2} \tilde{v}\right)-F\left(D^{2} u\right)\right| \leq C(\tilde{v}-u)+\frac{C_{3}(M)}{R}
$$

Now we apply Harnack's inequality for $\tilde{v}-u$ in $B\left(x_{0}, 4 M\right)$ (see (3.2)), to find

$$
\|\tilde{v}-u\|_{L^{\infty}\left(B\left(x_{0}, 3 M\right)\right)} \leq \frac{C_{4}(M)}{R} .
$$

The conclusion follows since $\left\|\tilde{v}-g_{0}\left(\left|x-y_{0}\right|-R\right)\right\|_{L^{\infty}\left(B\left(x_{0}, 3 M\right)\right)} \leq C(M) / R$.

Remark 1. From the proof of this Lemma, we can conclude that the statement remains true if we replace the assumption on the monotonicity of $u$ in the $e_{2}$ direction, with the hypothesis that

$$
B\left(y_{0}-t e_{2}, R\right) \subset\{u<0\} \text { for all } t \geq 0,
$$

and also, it suffices that

$$
\lim _{x_{2} \rightarrow-\infty} u\left(x_{1}, x_{2}\right)=-1, \quad \text { for }\left|x_{1}-y_{0} \cdot e_{1}\right| \leq 2 R .
$$

The next lemma proves the desired statement in Proposition 3.1, in the case when the distance between the centers is not arbitrarily large.

Lemma 3.3. If $B\left(y_{i}, R\right) \subset\{u<0\}, i=1,2$, and $\left|y_{1}-y_{2}\right| \leq R^{2}$, then the convex hull generated by $B\left(y_{i}, R-C \log R / R\right), i=1,2$, is included in $\{u<0\}$. 


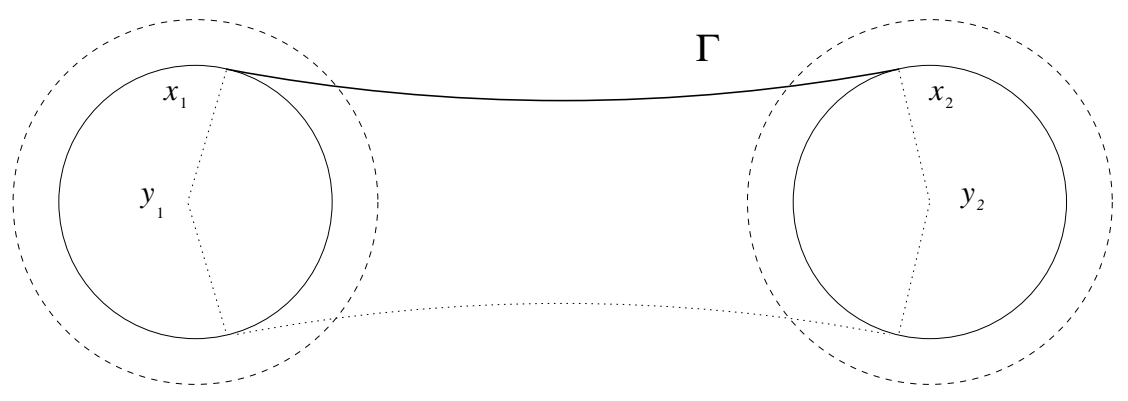

FiguRE 1.

Proof. Let $\Gamma$ be an arc of circle of radius $R^{5}$ tangent from above to the balls $B\left(y_{i}, R-\right.$ $C \log R / R)$ at $x_{i}, i=1,2$. (see Figure 1.)

Define,

$$
h_{\Gamma}(s)=\left(h_{0}+\frac{C}{R^{5}} \sigma\right)^{+}, \quad \sigma= \begin{cases}-1 & s \leq-1 / 2 \\ 2 s & |s| \leq 1 / 2 \\ 1 & s \geq 1 / 2 .\end{cases}
$$

Let $s_{\Gamma}$ be such that $h_{0}\left(s_{\Gamma}\right)=C / R^{5}$. We have that $s_{\Gamma} \sim c R^{-5 / 2}-1$, hence

$$
H_{\Gamma}:\left[c R^{-5 / 2}-1,1\right] \rightarrow \mathbb{R}, \quad\left|H_{\Gamma}\right| \leq C \log R,
$$

and therefore we can extend the corresponding $g_{\Gamma}=H_{\Gamma}^{-1}$ to be constant for $|t|>$ $C \log R$.

We want to estimate the supremum (maximum) of $H_{\Gamma}-H_{R}$. This occurs for the values of $s$ for which $h_{\Gamma}(s)=h_{R}(s)$. Using formulas (2.7) and (3.3) we find that the maximum is attained at values of $s$ with $1-|s|>c R^{-2}$. At such values we have, using (2.6),

$$
H_{\Gamma}(s) \leq H_{0}(s) \leq H_{R}(s)-\frac{C}{R} \log (1-|s|)<H_{R}(s)+\frac{c \log R}{R},
$$

which implies

$$
g_{\Gamma}\left(t+C \frac{\log R}{R}\right)>g_{R}(t), \text { for all } t \text { 's. }
$$

According to (2.5) , the function $v:=g_{\Gamma}\left(d_{\Gamma}\right)$, which is defined only for $x$ 's in a $C \log R$ neighborhood of $\Gamma$ (and with sides on the lines $l_{1}$ joining $x_{1}$ and $y_{1}$ and $l_{2}$ joining $x_{2}$ and $y_{2}$ ), is a strict supersolution. Also, on $l_{1}$, according to (3.4) we have

$$
v(x)=g_{\Gamma}\left(\left|x-y_{1}\right|-R+\frac{C \log R}{R}\right)>g_{R}\left(\left|x-y_{1}\right|-R\right) \geq u(x) .
$$

Analogously, $v \geq u$ on $l_{2}$. We claim that $v \geq u$ in the domain of definition of $v$. To see this, reflect $v$ across the line joining $y_{1}$ and $y_{2}$ and "glue" the two graphs by the 
constant function (see Figure 1). The new surface is a strict supersolution everywhere except on the sides, where it is above $u$. The conclusion follows by sliding this surface from $-\infty$.

We remark that the proof of Lemma 3.3 cannot be used to handle the case when the centers of the balls are far apart. Indeed, in this case any arc of circle $\Gamma$ "connecting" the two balls becomes too flat, and the corresponding supersolution $g_{\Gamma}\left(d_{\Gamma}\right)$ stops being on top of $u$ on the sides of its domain of definition. In the next Lemma, we overcome this difficulty, in the case when the two balls are centered on the same horizontal line.

Lemma 3.4. Assume $B(( \pm a, 0), R) \subset\{u<0\},(a>2 R)$. Then the convex hull generated by

$$
B\left(( \pm a, 0), R-C \frac{\log R}{R}\right)
$$

is contained in $\{u<0\}$.

Proof. Assume without loss of generality, that

$$
B\left(\left( \pm a,-R+C_{1} \frac{\log R}{R}\right), R\right) \subset\{u<0\} .
$$

Define the following convex function,

$$
k\left(x_{1}\right)=\frac{C_{2} \log \left(a+R-\left|x_{1}\right|\right)}{a+R-\left|x_{1}\right|}, \quad\left|x_{1}\right| \leq a .
$$

It is easy to check that

$$
k^{\prime \prime}\left(x_{1}\right) \geq \frac{C_{2} \log \left(a+R-\left|x_{1}\right|\right)}{2\left(a+R-\left|x_{1}\right|\right)^{3}} \text { and }\left|k\left(x_{1}\right)\right| \leq C_{2} \frac{\log R}{R} .
$$

Then, our claim follows if we prove that $\{u=0\}$ is above (in the $e_{2}$ direction) the graph of $k$.

We slide the graph of $k$ from $-\infty$, and denote with $z_{1}$ the first coordinate of the first touching point with $\{u=0\}$. Denote by $k_{t}$ the $t$-translate downward in the $e_{2}$ direction, for which the first touching point occurs. We start by proving that $z_{1}$ cannot occur well-inside the interval $[-a, a]$.

Assume $d:=a-\left|z_{1}\right| \geq R$, then the tangent line to the graph of $k_{t}$ at $z_{1}$ separates from the graph at $z_{1} \pm d / 2$ a distance

$$
\left[\min _{\left|t-z_{1}\right| \leq \frac{d}{2}} k^{\prime \prime}(t)\right] \frac{d^{2}}{8} \geq m C_{2} \frac{\log d}{d}
$$

with $m$ a fixed number. Therefore, if $C_{2}$ is large then $\left(z_{1}, k\left(z_{1}\right)\right)$ belongs to the convex hull generated by

$$
B\left(w_{i}, \frac{d}{20}-C \frac{\log d}{d}\right), i=1,2
$$


where $B\left(w_{i}, d / 20\right)$ are tangent balls to the graph of $k_{t}$ with centers $w_{i}$ on the lines $x_{1}=z_{1} \pm d / 2$. However, according to Lemma 3.3, such convex-hull is contained in the negative side of $u$. Thus, $\{u=0\}$ cannot be tangent to (and above) the graph of $k$ at $z_{1}$, with $\left|z_{1}\right| \leq a-R$.

Now, we show that $z_{1}$ cannot occur near the sides either, until the graph of $k_{t}$ coincides with the graph of $k$. To see this, assume $z_{1}>0$, and let $l$ be the line passing through $\left(a-R, k_{t}(a-R)\right)$ and with slope equal to the slope of $k$ at $a$. Again, $l$ will separate from the graph of $k_{t}$ at $a-2 R$ a distance comparable to $C_{2} \log R / R$. Thus, for $C_{1}$ and $C_{2}$ large enough, the graph of $k_{t}$, for $z_{1} \in[a-R, a]$, will be contained in the convex hull generated by

$$
B\left(p, \frac{R}{2}-C \frac{\log R}{R}\right) \quad \text { and } \quad B\left(\left(a,-\frac{R}{2}+C_{1} \frac{\log R}{R}-t\right), \frac{R}{2}-C \frac{\log R}{R}\right) \subset\{u<0\},
$$

where $B(p, R / 2)$ is the tangent ball to the graph of $k_{t}$ with center on the line $x_{1}=$ $a-2 R$. According to Lemma 3.3 this set is contained in the negative side of $u$, hence so is the graph of $k_{t}$ on $[a-R, a]$.

Corollary 3.5. If $B\left(y, C_{0}\right), B\left(z, C_{0}\right) \subset\{u<0\}$, for a large constant $C_{0}$, then

$$
\left\{\left(x_{1}, x_{2}\right): x_{2} \leq \min \left\{y_{2}, z_{2}\right\}, \quad x_{1} \in\left[y_{1}, z_{1}\right]\right\} \subset\{u<0\} .
$$

Corollary 3.5 follows immediately by using the monotonicity of $u$ and sliding the ball at greatest height at the level $\min \left\{y_{2}, z_{2}\right\}$. Then, apply Lemma 3.4 .

Remark 2. As in Remark 1, in the statement of Lemma 3.4 we can replace the assumption on the monotonicity of $u$ in the $e_{2}$ direction, with the hypothesis that

$$
B(( \pm a,-t), R) \subset\{u<0\} \quad \text { for all } t \geq 0,
$$

and also it suffices that

$$
\lim _{x_{2} \rightarrow-\infty} u\left(x_{1}, x_{2}\right)=-1, \quad \text { for }\left|x_{1}\right| \leq a+2 R .
$$

Remark 3. If $B\left(y_{i}, R\right) \subset\{u<0\}, i=1,2$ and the centers do not belong to the same horizontal line, then we cannot use the argument in Lemma 3.4 to reach the same conclusion.

Indeed, let $\nu$ be the unit vector perpendicular to $\overline{y_{1} y_{2}}$, and assume $\nu \cdot e_{2} \geq \alpha>0$. Define the corresponding function $k$ in the coordinate system $\left(\nu, \nu^{\perp}\right)$ "connecting" the two balls $B\left(y_{i}, R-C_{1} \log R / R\right)$. By sliding the graph of $k$ in the $e_{2}$ direction, at the first contact point, we cannot guarantee that tangent balls to the graph of $k$ will be contained in the negative side of $u$ (see Figure 2).

However, if we modify the radii of the balls $B\left(w_{i}, d / 20\right)$ by a factor depending on $\alpha$, this would guarantee that they are below the graph of $k$ in the $e_{2}$ direction, and therefore below the graph of $u$.

This, let us conclude that the convex hull generated by $B\left(y_{i}, R-C(\alpha) \log R / R\right)$ is included in $\{u<0\}$, with $C(\alpha) \rightarrow \infty$ as $\alpha \rightarrow 0$. 


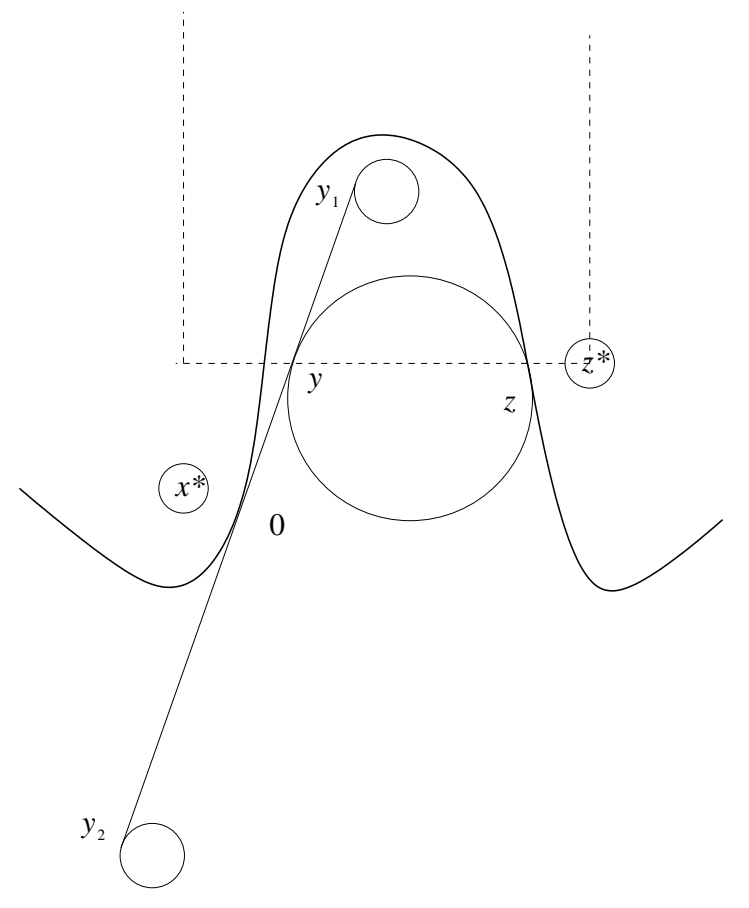

FiguRE 2.

In order to prove a stronger result than the one in Remark 3, we show the following.

Lemma 3.6. (Non-oscillation of the zero set.) Let $\nu$ be a unit vector such that $\nu \cdot e_{2}>0$. Assume that the segment $\overline{y_{1} y_{2}}:=\left\{t \nu^{\perp},|t| \leq d\right\}$ is included in $\{u \leq 0\}$ and tangent to $\{u=0\}$ at 0 . If $B\left(y_{i}-C \nu, C\right) \subset\{u<0\}, i=1,2$, for some $C$ large enough, then

$$
B\left(t \nu^{\perp}-\frac{d}{6} \nu, \frac{d}{6}\right) \subset\{u<0\}, \quad \text { for all }|t| \leq 2 d / 3 .
$$

Proof. Without loss of generality, we assume that (see Figure 2)

$$
\left(y_{1}-y_{2}\right) \cdot e_{2}>0
$$

Since $B\left(y_{i}-C \nu, C\right) \subset\{u<0\}$, we have that,

$$
S:=\left\{t \nu^{\perp}-r \nu,|t| \leq d, 0 \leq r \leq C\right\} \subset\{u<0\} .
$$

Let $y=t \nu^{\perp},|t| \leq 2 d / 3$. Consider the ball $B_{r}$ of radius $r$, tangent to $\overline{y_{1} y_{2}}$ at $y$ from below. From (3.6), we find that for $r<C / 2, B_{r} \subset\{u<0\}$. Now, we increase $r$ till $B_{r}$ touches $\{u=0\}$ for the first time outside the strip $S$. We need to show that $r \leq d / 6$.

Assume not, i.e. $\frac{C}{2} \leq r \leq \frac{d}{6}$ and denote with $z$ the tangent point of $B_{r}$ and $\{u=0\}$ (see Figure 2). 
According to (3.6), we also know that $B(-C \nu / 2, C / 2) \subset\{u<0\}$ is tangent to $\{u=0\}$ at 0 . From Lemma 3.2 we find points $x^{*}$ and $z^{*}$ at finite distance from 0 and $z$ such that $B\left(x^{*}, c_{0}\right), B\left(z^{*}, c_{0}\right) \subset\{u>0\},\left(\left|x^{*}\right|,\left|z^{*}-z\right|\right.$ of order $\left.1 / c_{0}\right)$ where $c_{0}$ is a given constant, all provided that $C$ (hence $r$ ) is large. By Corollary 3.5 (applied upside-down) we find that $u$ is strictly positive in the set

$$
\left\{\left(x_{1}, x_{2}\right): x_{2} \geq \max \left\{z_{2}^{*}, x_{2}^{*}\right\}, \quad x_{1} \in\left[z_{1}^{*}, x_{1}^{*}\right]\right\} .
$$

By simple geometrical considerations we find that $y_{1}$ belongs to such set, and we obtain a contradiction (see Figure 2).

Now, combining the techniques of Lemma 3.4 together with Lemma 3.6, we will deduce the desired Proposition 3.1.

Proof of Proposition 3.1. We refer to the notation of Lemma 3.4.

Denote by $D$ the closed region bounded by the balls $B\left(y_{i}, R\right), i=1,2$ and the corresponding graphs of $k$ connecting them (from "above" and "below"). Our conclusion follows if we prove that $D$ is contained in the negative side.

We slide $D$ from $-\infty$. By performing a change of coordinates, we can assume that we are in the same setting as in Lemma 3.4, that is

$$
\begin{aligned}
& B\left(\left( \pm a,-R+C_{1} \frac{\log R}{R}\right), R\right) \subset\{u<0\}, \\
& k\left(x_{1}\right)=\frac{C_{2} \log \left(a+R-\left|x_{1}\right|\right)}{a+R-\left|x_{1}\right|}, \quad\left|x_{1}\right| \leq a,
\end{aligned}
$$

with the direction of monotonicity not necessarily equal to $e_{2}$.

It suffices to show that if the region $D_{t}$ between the graph of $k$ and its reflection across the line $x_{2}=-R+C_{1} \log R / R$ is included in $\{u \leq 0\}$, then $\{u=0\}$ cannot be tangent to $D_{t}$ at a point $z=\left(z_{1}, k\left(z_{1}\right)\right)$.

In the case when $\left|z_{1}\right|>a-R$, the contradiction follows as in Lemma 3.4, since $B(p, R / 2) \subset D_{t}$.

If $d:=a-\left|z_{1}\right| \geq R$, let $l$ be the tangent segment at $z$ to the graph of $k$ between the lines $x_{1}=z_{1} \pm d / 2$. Then, $l$ satisfies the assumptions of Lemma 3.6. The existence of small balls tangent to $l$ at the endpoints and contained in the negative side, follows from the fact that $D_{t} \subset\{u \leq 0\}$. Therefore the balls

$$
B^{q_{i}}:=B\left(q_{i}, d / 12\right), i=1,2,
$$

tangent to $l$ and with centers on the lines $x_{1}=z_{1} \pm(d / 4)$ are included in $\{u<0\}$.

As in Lemma 3.4, we consider the balls

$$
B^{w_{i}}:=B\left(w_{i}, d / 20\right), i=1,2,
$$

tangent to the graph of $k$, and with centers on the line $x_{1}=z_{1} \pm d / 4$ respectively. To conclude the proof it suffices to show that $B^{w_{i}} \subset\{u<0\}$. We will show that

$$
B^{w_{i}} \subset D_{t} \cup B^{q_{i}}
$$


Notice that the balls $B^{w_{i}}$ and $B^{q_{i}}$ with centers on the same vertical line, are below $x_{2}=C_{3} \log d / d$ and intersect $x_{2}=-C_{3} \log d / d$. This implies that $B^{w_{i}} \backslash B^{q_{i}}$ is included in $\left|x_{2}\right| \leq C_{4} \log d / d$, which proves (3.7).

As an immediate consequence, we obtain the following statement.

Corollary 3.7. There exists a convex set $K \subset\{u<0\}$ such that $B\left(x, C_{0}\right) \cap\{u=$ $0\} \neq \emptyset$ for all $x \in \partial K$ and $C_{0}$ large.

Proof. Choose $R=C_{0}$ large such that $R-C \log R / R>0$, and consider all $y$ 's such that $B(y, R) \subset\{u<0\}$. The convex hull generated by such $y$ 's satisfies the required properties.

Lemma 3.8. The set $K$ is a half-plane.

Proof. Assume by contradiction that $K$ is not a half-plane. Then there is a line $l$ tangent to $K$, say at 0 , such that $\partial K$ separates at least linearly from $l$ at $\pm \infty$. Thus we can find tangent balls of arbitrarily large radius $R$ tangent to $\{u=0\}$ at $x_{+}, x_{-}$ below the line $l-M e_{2}$, for any large $M$. From Lemma 3.2, there exist balls of radius $C_{0}$ on the positive side and below $l$ near $x_{+}$and $x_{-}$(provided that $R$ and $M$ are large.) We apply Proposition 3.1 and obtain that the line connecting the centers of these balls is included in $\{u>0\}$, and we reach a contradiction since it also intersects $K$.

As a consequence of Lemma 3.8 and its counterpart in the positive side, we conclude that $\{u=0\}$ is contained in a strip. Next, we show that the width of such strip is arbitrarily small, hence $\{u=0\}$ is a line, which concludes the proof of Theorem [1.1.

Proof of Theorem 1.1. Assume

$$
\{u=0\} \subset\{0 \leq x \cdot \nu \leq d\},
$$

and $u\left(\frac{1}{M} \nu\right)>0$, for some direction $\nu$ such that $\nu \cdot e_{2}>0$. By taking a large ball of radius $R$ tangent to $\{u=0\}$ from below and centered on the line $t \nu$, we find a ball $B_{M}$ of radius $M$ at distance at most $2 / M$ from $\{x \cdot \nu=0\}$, which is included in $\{u>0\}$. By applying Proposition 3.1 to the ball $B_{M}$ and all the balls of radius $M$ tangent from above to $\{x \cdot \nu=d\}$ we find that $\{u=0\}$ is below

$$
x \cdot \nu=\frac{2}{M}+\frac{C \log M}{M}<\frac{d}{2}
$$

for $M$ large.

We now wish to prove that the limit assumption (3.1) holds true.

Let $v$ be any viscosity solution to (1.2), then there exists $0<\alpha<1$, such that the $C^{\alpha}$ norm of $v$ is bounded by a universal constant (see [2]). Thus, if $u$ is as in Theorem 1.1, then family of solutions $u_{t}(x):=u\left(x+t e_{2}\right)$ to (1.2) will converge uniformly to a function $g_{ \pm}$, as $t \rightarrow \pm \infty$, or equivalently

$$
\lim _{x_{2} \rightarrow \pm \infty} u\left(x_{1}, x_{2}\right)=g_{ \pm}\left(x_{1}\right)
$$


with $g_{ \pm}$one-dimensional viscosity solution to the same equation (1.2). Precisely, since $g_{ \pm}$is one-dimensional, it satisfies in the viscosity sense the following equation

$$
g^{\prime \prime}=h_{0}^{\prime}(g)
$$

Hence, $g_{ \pm}$is a classical solution to (3.8). Moreover, since $|u| \leq 1$, then $\left|g_{ \pm}\right| \leq 1$ as well, and $g_{-} \leq g_{+}$. We proceed to classify all possible solutions $g$ to (3.8).

Notice that

$$
\lambda \leq h_{0}^{\prime} / f \leq \Lambda
$$

hence according to (1.4) $h_{0}^{\prime}>0$ on $\left(-1, s_{0}\right)$ and $h_{0}^{\prime}<0$ on $\left(s_{0}, 1\right)$, where $s_{0}$ is the unique zero of $f$ in $(-1,1)$. Therefore, the only constant solutions to (3.8) are $g \equiv \pm 1$ and $g \equiv s_{0}$. If $g$ is not identically constant, then as in Section 2, we define the function $h$ by the relation

$$
g^{\prime}(t)= \pm \sqrt{2 h(g(t))}
$$

on a maximal interval $I$ on which $g$ is increasing or decreasing.

Then,

$$
g^{\prime \prime}(t)=h^{\prime}(g(t)) .
$$

Combining (3.8) with (3.9) we obtain that

$$
h^{\prime}(s)=h_{0}^{\prime}(s), s \in g(I)=(a, b),
$$

and

$$
h(a)=h(b)=0 .
$$

From this, we deduce that

$$
h=h_{0}-h_{0}(a)=h_{0}-h_{0}(b) .
$$

If $(a, b)$ is strictly contained in $(-1,1)$, then $g$ is periodic (of period $2(H(b)-H(a))$ ). Also the constant solution $g=s_{0}$ can be interpreted as a limit of periodic solutions as the interval $(a, b)$ tends to $s_{0}$.

If $(a, b)=(-1,1)$ then, $g$ coincides (up to a translation) with the one-dimensional solution $g_{0}$ or with its the reflection across the vertical axis through zero.

In the case when $g_{+}$is periodic, then since $g_{-}<g_{+}, g_{-}$can only be the constant solution -1 . As in Lemma 2.4 we construct a radial function $g_{\rho}$ which is a supersolution everywhere except on a level set above the range of $g_{+}$, and therefore above $u$. Moreover, the "profile" of $g_{\rho}$ is a perturbation of $g_{0}$. By sliding this supersolution from $-\infty$ we find that $u \leq \min g_{\rho}$, thus $g_{+} \leq \min g_{\rho}$ and we reach a contradiction.

In the case when $g_{+}$is the the one-dimensional solution $g_{0}$, then $\{u<0\} \subset\left\{x_{1}<\right.$ $0\}$. Since $g_{-}<g_{+}$, there exist $\delta$ and $M$ such that

$$
\left\{0<x_{1}<\delta, x_{2}<-M\right\} \subset\{u<0\} .
$$

Therefore, we can find a ball $B_{R}$ of radius $R$ arbitrarily large, tangent to the line $x_{1}=\delta / 2$ included in $\{u<0\} \cap\left\{x_{1} \leq \delta / 2\right\}$. We apply Remark 2 to $B_{R}$ and all the balls of radius $R$ tangent to $x_{2}=0$ from the negative side, and obtain that 


$$
\left\{x_{1}<\delta / 2-C \log R / R\right\} \subset\{u<0\} .
$$

Thus, $g_{+} \leq 0$ in $\left\{x_{1}<\delta / 4\right\}$ for $R$ large enough, which is a contradiction.

Hence, we conclude that $g_{+} \equiv+1$, and similarly $g_{-} \equiv-1$.

\section{REFERENCES}

[1] Ambrosio L., Cabre X., Entire solutions of semilinear elliptic equations in $\mathbb{R}^{3}$ and a conjecture of De Giorgi, J. American Math. Soc. 13 (2000), 725-739.

[2] Caffarelli L., Cabre X., Fully Nonlinear Elliptic Equations, Colloquium Publications 43, American Mathematical Society, Providence, RI, 1995.

[3] Ghoussoub N., Gui C., On a conjecture of De Giorgi and some related problems, Math. Ann. 311 (1998), 481-491.

[4] Savin O., Regularity of flat level sets for phase transitions. Ann. of Math. (to appear).

Department of Mathematics, Johns Hopkins University, Baltimore, MD 21218

E-mail address: desilva@math.jhu.edu

Department of Mathematics, Columbia University, New York, NY 10027

E-mail address: savin@math.columbia.edu 